\title{
Chapter 1 \\ Preferential concentration of finite solid particles in a swirling von Kármán flow of water
}

Martin Obligado, Romain Volk, Nicolas Mordant and Mickael Bourgoin

\subsection{Introduction}

Turbulent flows laden with inertial particles are widely encountered in nature (particles dispersion in the atmosphere, rain formation, marine snow sedimentation...) and in industry (fuel or coal combustion, fluidized beds reactors, separation techniques...). In all these configurations, inertial particles interacting with turbulence form high and low concentration regions leading to non-trivial spatial organization of particles: this so-called preferential concentration. This phenomenon involves many ingredients such as particle inertia, turbulence characteristics, gravitational settling, disperse phase volume fraction, and many others. The specific role of each parameter remains to be clarified (a review on this topic can be found on [7]). It is in this context that the study of preferential concentration of particles in different types of flows is important, as it allows to isolate the role of each parameter.

Most numerical studies of preferential concentration have considered the case of high density ratios of particles using purely stokesian point particle models in direct numerical simulations of turbulence. In such conditions particles inertia is primarily quantified by the Stokes number, $S t=\tau_{p} / \tau_{\eta}$ (with $\tau_{p}$ the particles relaxation time and $\tau_{\eta}$ the turbulent dissipation scale). Experimental counter-part studies have

Martin Obligado

Univ. Grenoble Alpes, CNRS, Grenoble INP*, LEGI, 38000 Grenoble, France. e-mail: martin.obligado@univ-grenoble-alpes.fr

Romain Volk

Laboratoire de Physique de l'École Normale Supérieure de Lyon, CNRS \& Université de Lyon, 46 allée dItalie, F-69364 Lyon Cedex 07, France. e-mail: romain.volk@ens-lyon.fr

Nicolas Mordant

Univ. Grenoble Alpes, CNRS, Grenoble INP*, LEGI, 38000 Grenoble, France. e-mail: nicolas.mordant@univ-grenoble-alpes.fr

Mickael Bourgoin

Laboratoire de Physique de l'École Normale Supérieure de Lyon, CNRS \& Université de Lyon, 46 allée dItalie, F-69364 Lyon Cedex 07, France. e-mail: mickael.bourgoin@ens-lyon.fr 
investigated for instance the preferential concentration of small (sub-kolmogorov) water droplets in grid-generated wind-tunnels (see for instance [11]). These studies show qualitative agreements between experiments and numerics, for instance with a maximum of clustering around Stokes numbers of order unity. However a quantitative systematic comparison remains to be carried, accounting in particular for the role of gravity (not always included in numerical studies) and for trends with Reynolds number (which were found to be quite dramatic in experiments [11]), in order to clarify the exact mechanism at play and leading to preferential concentration (for instance in terms of centrifugation [15] or sweep-stick [4]).

The case of moderate density ratios remains less investigated, particularly in experiments. Fiabane and collaborators characterized the clustering properties of finite size neutrally buoyant particles and glass particles in water and homogeneous isotropic turbulence [2] at Reynolds number up to $R_{\lambda} \approx 400$ (based on Taylor microscale). Their study shows in particular that in this case the Stokes is not any longer a relevant indicator of clustering.

Here we present a study of preferential concentration with Voronoï diagrams of solid particles at moderate density ration in a von Kármán flow of water at a Reynolds number up to $R_{\lambda} \approx 810$. We investigate preferential concentration of PMMA particles with density $\rho_{p}=1400 \mathrm{~kg} / \mathrm{m}^{3}$ and diameter $2.8<d_{p} / \eta<6.3$ for $340<R e_{\lambda}<810$. Therefore, with high $R e_{\lambda}$ and heavy particles in the a range of diameters close to the limit classically considered for point particle dynamics, i.e. around $d_{p} / \eta \sim 5 \eta[2]$.

\subsection{Experimental setup.}

The flow is a turbulent von Kármán swirling flow, described in references [12, 13]. Water fills a cylindrical container of internal diameter $D=15 \mathrm{~cm}$ and a length of $25 \mathrm{~cm}$. It is driven by two disks of diameter $14 \mathrm{~cm}$, fitted with blades. Three different rotation rates were studied: 2.85 .6 and $8.3 \mathrm{~Hz}$ (figure 1.1a). The distance between the disks is $20 \mathrm{~cm}$ while the rotation frequency is imposed by two calibrated constant current engines. The angular velocity is measured with two dynamos with a precision in the order of $2 \%$ and is adjusted in a way that the disks have the same velocity but opposite direction (figure 1.1b). This setup allows us to obtain developed turbulence with $R e_{\lambda}$ up to $\sim 800$ in a compact region of space. This type of flow has been extensively used for studying the properties of turbulence, Lagrangian $([5,8,14])$ or Eulerian ([16]).

The von Kármán flow generated has a large scale non-homogeneous, nonisotropic 3D mean structure (figure 1.1b). As the disks counter-rotate, the mean flow has a strong azimuthal velocity component (in the order of $\pi D \Omega$ close to the disks) and vanishes in the middle plane of the cylinder. Furthermore, the impeller disks work as centrifugal pumps ejecting the flow in the radial direction near the disks, creating a poloidal re-circulation with a stagnation point at the center of the cylinder. 
The turbulence generated is locally homogeneous in the center of the cylinder but conserves the anisotropy for big and small scales ([14]). In the limit $R e_{\lambda}>>1$ the usual local isotropy hypothesis is generally assumed for the small scales of the flow (although the validity of this assumption has been recently questioned [10]). For the measurements reported here, the Taylor-based Reynolds number reaches values up to 810 and the dissipation rate $\varepsilon$ values up to $25 \mathrm{~W} / \mathrm{kg}$ (table 1.1 ). We study PMMA particles with density ratio 1.4 and two different radii: 80 and $100 \mu \mathrm{m}$. The water temperature was maintained constant during all measurements at $T=30^{\circ} \mathrm{C}$. As the flow is anisotropic, the Reynolds number based in the Taylor scale is estimated as $R e_{\lambda}=\sqrt{\frac{15 u^{4}}{\varepsilon v}}$, where $u=\sqrt{\frac{2 u_{x}^{2}+u_{2}^{2}}{3}}$, is obtained using the rms value of velocity fluctuations .

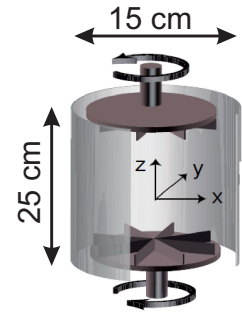

a

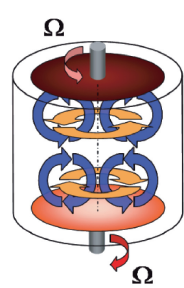

b

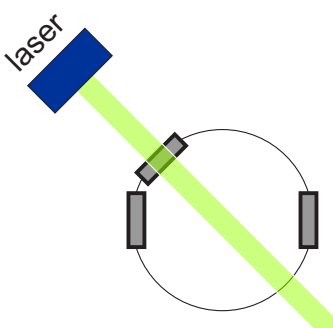

camera 1

C

Fig. 1.1 (a) Schema of the experimental setup. (b) Inhomogeneous flow generated in the container. (c) Schema of the imaging system.

The cylindrical container is equipped with three windows that allow a proper visualization of the flow. The flow passes through a laser sheet (inclined $45^{\circ}$, as shown in figure 1.1c), where a high-speed camera Phantom-Miro M310 was placed in the horizontal direction. The laser produces a continuous beam with a wavelength of $532 \mathrm{~nm}$ working at $5.2 \mathrm{~W}$. The camera is connected to a Scheimpflug system in order to have a proper focus of the measurement section.

\begin{tabular}{|c|c|c|c|c|c|c|}
\hline$\Omega(\mathrm{Hz})$ & $u_{x}(\mathrm{~m} / \mathrm{s})$ & $u_{z}(\mathrm{~m} / \mathrm{s})$ & $\eta(\mu \mathrm{m})$ & $\tau_{\eta}(\mathrm{ms})$ & $\varepsilon\left(\mathrm{Wkg}^{-1}\right)$ & $R e_{\lambda}$ \\
\hline \hline 2.8 & 0.38 & 0.19 & 28.5 & 3.55 & 3.1 & 340 \\
\hline 5.6 & 0.76 & 0.41 & 21.2 & 7.11 & 7.2 & 570 \\
\hline 8.3 & 1.14 & 0.62 & 16.4 & 20.7 & 20.7 & 810 \\
\hline
\end{tabular}

Table 1.1 Turbulent parameters of the flow for the three velocities studied. The values are obtained using the results in [13].

10 films are recorded for each configuration (i.e. for each given particle class and rotation rate of the impellers) with a sample rate of $3200 \mathrm{fps}$ at a resolution of 
\begin{tabular}{|l|l|l|l|l|}
\hline Particle & $d_{p}(\mu \mathrm{m})$ & $S t_{\Omega 1}$ & $S t_{\Omega 2}$ & $S t_{\Omega 3}$ \\
\hline
\end{tabular}

\begin{tabular}{|c|c|c|c|c|}
\hline \hline 1 & 80 & 0.83 & 1.50 & 2.50 \\
\hline 2 & 100 & 1.30 & 2.30 & 4.0 \\
\hline
\end{tabular}

Table 1.2 Particles considered and their Stokes number for each velocity.

$1280 \times 800$ at 12 bits (which considering camera's available internal memory gives 8309 images per film, being $\sim 2.60 \mathrm{~s}$ ). Therefore, $\sim 26 \mathrm{~s}$ were recorded for granting proper statistical accuracy. The real size of the window measured in the real world is $\sim 4 \times 2 \mathrm{~cm}^{2}$. A calibration mask was used for performing pixel to real world transformation.

\subsection{Results}

Once the films are recorded, particles centers can be identified with sub-pixel accuracy, by using standard tracking techniques. Then, Voronoï tessellations can be obtained, following [6]. Figure 1.2 shows the probability density functions (PDF) of the normalised Voronoï area $\mathscr{V}=A / \bar{A}$ (with $A$ the area of a Voronoï cell and $\bar{A}$ their average) and of $\log (\mathscr{V})$ centered and reduced.
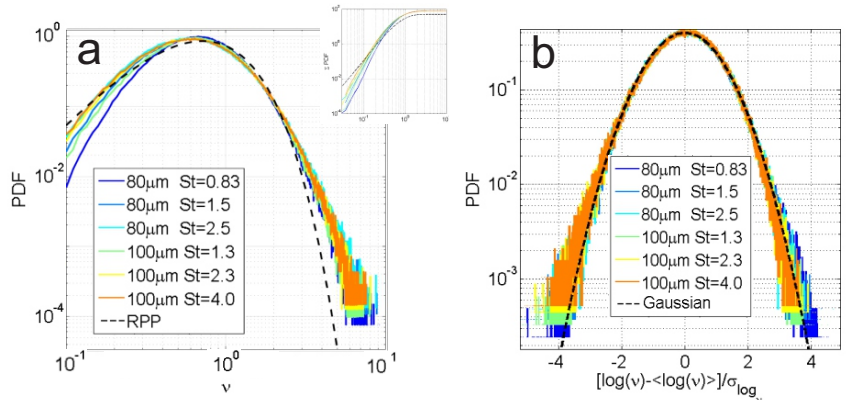

Fig. 1.2 (a) PDF of normalized Voronoï areas $\mathscr{V}$ for experiments at different Reynolds number and Stokes number. The black dashed line represents a random Poisson process (RPP) distribution. (b) PDF, centered and reduced, of $\log (\mathscr{V})$. The black dashed line represents a Gaussian distribution.

It can be clearly seen that the measured distributions are not that of a uniform random process (figure 1.2a). Large Voronoï areas are significantly over-represented compared to the RPP case, indicating the existence of large depleted regions. Similarly, normalized areas between a few tenth (depending on the particle class) and about $\mathscr{V} \sim 0.5$ are over-represented in the experiment compared to a RPP, indicating the preferential concentration phenomenon. Besides, the most probable value for the normalized Voronoï area is smaller for the particles than for a RPP, which 
indicates the predominance of over-concentrated areas. Much smaller areas are on the contrary under-represented. However, the log-log representation misleadingly exacerbates this under-representation. As shown in the inset in figure 1.2a, showing the cumulative PDF, normalized areas above $0.15-0.3$ are already cumulatively more represented with the inertial particles compared to a RPP.

Big Voronoï areas (or depleted regions) are robust when changing particle and flow parameters, while small areas (or concentrated regions) are affected by these changes. This has been already observed in the previous study by Monchaux and collaborators [6] at moderate Reynolds number. In that work it was also observed that the Voronoï area PDFs for inertial particles in turbulence were well approximated by a lognormal distribution. This is confirmed as a robust characteristic also preserved in the higher Reynolds number regime explored in the present study, as it can be seen in figure $1.2 \mathrm{~b}$ : the PDF of $\log (\mathscr{V})$ is approximately Gaussian, at least within the range $\pm 3 \sigma_{\log (\mathscr{V})}$. Deviation from lognormality is only observed for small values of $\log (\mathscr{V})$ which are slightly over-estimated. Therefore, the overall statistical distribution of Voronoï areas is almost characterized entirely by one single parameter, which we choose to be the standard deviation $\sigma_{\mathscr{V}}$ and whose dependency with experimental parameters can be used to quantify the evolution of particles clustering.
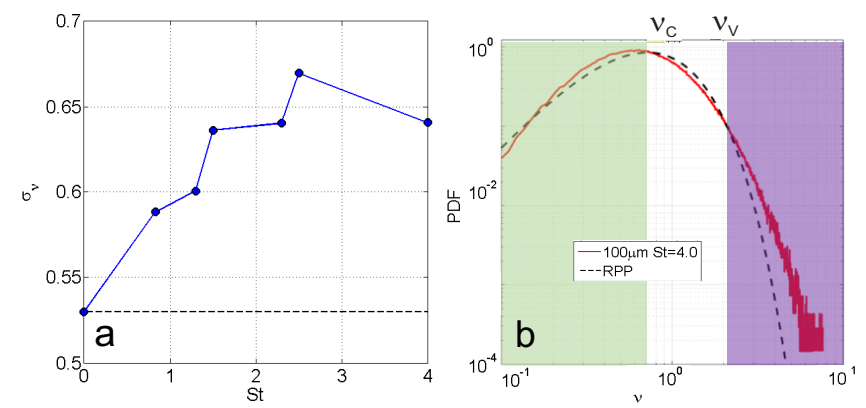

Fig. 1.3 (a) Standard deviation of normalized Voronoï areas $\sigma_{\mathscr{V}}$ as a function of the Stokes number. The point for $S t=0$ has been artificially added while the black dashed line represents the value $\sigma_{\mathscr{V}}=0.53$, that correspond to a RPP phenomenon. (b) Schema of clusters and voids detection.

Figure 1.3a shows the standard deviation of normalized Voronoï areas $\sigma_{\mathscr{V}}$ as a function of Stokes number. It can be observed that it is clearly larger than $\sigma_{\mathscr{V}}=0.53$, that corresponds to a RPP. This is a strong evidence of the formation of clusters and voids in our system, while the amount of clustering tends to grow when $S t$ is increased with possibly a saturation or even a reduction for the largest explored Stokes number. This may suggest a maximum of clustering for $S t \sim 3-4$, as also observed by Monchaux et al., although the available data here does not allow to be conclusive on this point. 


\subsubsection{Clusters}

In the present section, we will focus on the identification and characterisation of clusters and voids formed by the particles. One important aspect concerns the analysis of the geometry of the clusters. Do they have a fractal structure? Do they have a characteristic size? As voids presents similar properties, we will only present results from the clusters study (a similar analysis for the voids on this experiment can be found in [9]).

To define a cluster and a void, we follow the idea proposed by Monchaux et al. $[6,7]$. Clusters and voids are easily identified by choosing an appropriate threshold for particles local concentration (defined as the inverse of Voronoï cell area) as can be seen in figure 1.3b (where the distribution for higher $S t$ of figure 1.2 is shown). The two intersections of the experimental PDF with the RPP closer to the maximum of the distribution are used as thresholds. The values that satisfy $\mathscr{V}<\mathscr{V}_{C}$ are defined as clusters while the values where $\mathscr{V}>\mathscr{V}_{V}$ as voids. The values in between are not considered clusters nor voids. The referred work shows the validity of $\mathscr{V}_{C}$ and $\mathscr{V}_{V}$ as such thresholds. These two parameters are robust when varying $S t$ and $R e_{\lambda}$, as it can be observed in figure 1.2a. The resulting process for obtaining clusters and voids from the centers in real world is shown in figure 1.4.
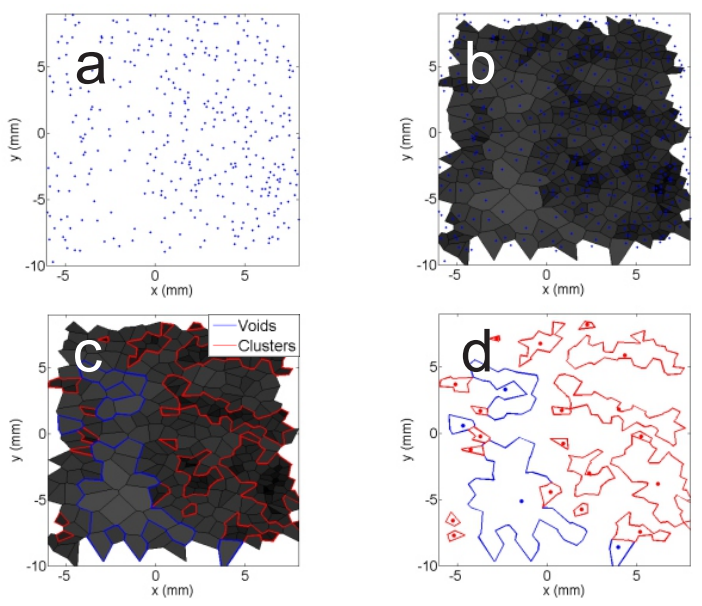

Fig. 1.4 Schema of clusters and voids detection. Starting from particle's centers in real space (a), the Voronoï diagrams are obtained (b). With the method explained above, clusters and voids are identified (c). In a final step, centers of mass of clusters and voids are calculated (d).

Once clusters have been identified, their geometry can be analysed. Figure 1.5a shows the clusters area PDFs, normalised by the mean area value. Surprisingly, all curves collapse and show a clear peak at $\frac{A_{V}^{\max }}{\left\langle A_{C}\right\rangle} \sim 0.15$. This is a strong experimental evidence of a system with a typical cluster area. We also observe a power law be- 
haviour of area PDF for large events, with an exponent of the order of $-5 / 3$, slightly less steep than the -2 exponent reported by Monchaux et al., but in good agreement with [11] and the predictions from [3]. Figure 1.5b shows how the mean value of clusters area varies with $S t$. This value tends to grow with $S t$, and it seems to reach an asymptotic value of the order of $3.5 \mathrm{~mm}^{2}$ (corresponding to $\sim 100 \eta^{2}$ ) for $S t \sim 4$, although more values would be required to confirm this asymptotic trend.
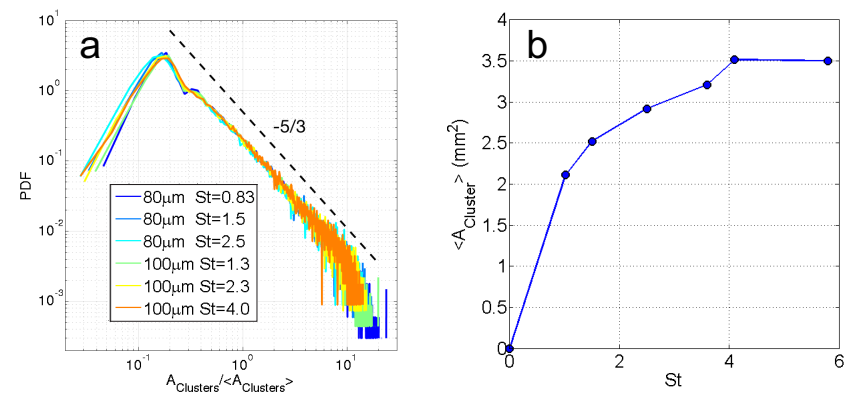

Fig. 1.5 (a) Clusters area PDFs, normalized by the mean area value. (b) Mean value of clusters area as a function of Stokes number.

Aliseda and collaborators [1], working with grid turbulence in a wind tunnel with $R e_{\lambda}=75$, using qualitative inspection found that poly-dispersed water droplets form clusters with a typical area of $10 \eta$. In order to compare results, figure 1.6a shows $\sqrt{\left\langle A_{C}\right\rangle} / \eta$ as a function of $R e_{\lambda}$ and $S t$. Figure 1.6b shows the same relationship but considering the most probable cluster area $A_{C}^{\max }$ instead of the average area $<$ $A_{C}>$ (i.e. the peak of the PDFs of areas in figure 1.5a). The fact that the PDF of clusters area collapses, means that the mean area and the most probable area are simply related by a proportionality relation: $\left.A_{C}^{\max }=C<A_{C}\right\rangle$ with $C=0.15$ for clusters. Therefore, both curves in figure 1.6 show a similar trend.

Interestingly, the characteristic areas reported here are always bigger than the results reported in [1], the minimum value reaches $\sqrt{A_{C}^{\max }} / \eta=20$, while the maximum goes up to $50 \eta$ (figure 1.6c). Such typical dimensions of clusters are larger than previously reported values in experiments with small droplets at larger density ratios (such as [11]) at similar St and Reynolds numbers. The trends are however qualitatively consistent. In particular, Sumbekova et al. reported a strong dependency of cluster size with Reynolds number, in agreement with the present observations. Note that in our experiment it is difficult though to clearly disentangle Stokes and Reynolds number dependencies (what would require to systematically vary the actual particle diameters).

Finally, figure 1.6d shows cluster perimeters as a function of the square root of its area. The figure shows many different tendencies but the fractional behavior of the exponent evidences the fractal nature of clusters with the presence of several different populations. Moreover, the almost continuum range for this exponent (ranging from $\sim 1.4$ to $\sim 3.5$ ) and the differences with previous works evidence the extreme 

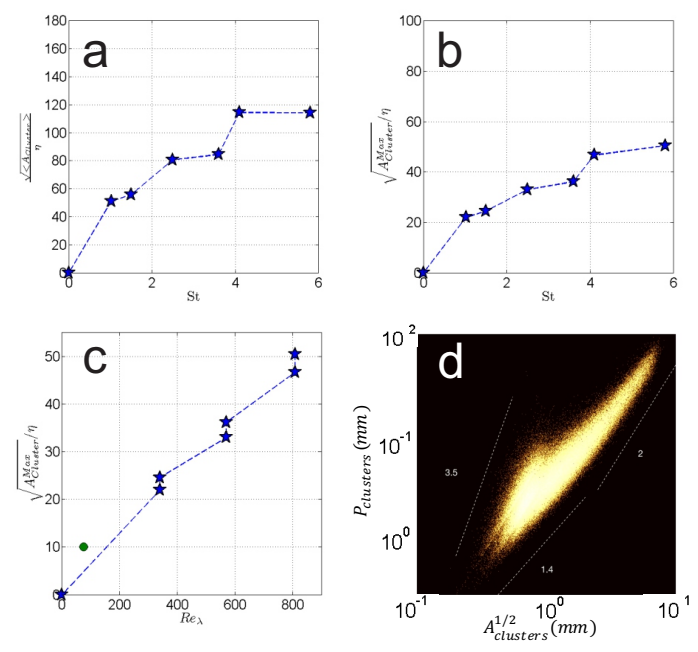

Fig. 1.6 (a) Square root of the mean value of clusters area, normalized with the Kolmogorov length scale $\eta$ as a function of Stokes number. (b) Same figure as before but for the peak of cluster area distribution. (c) Value of the peak of cluster area distribution as a function of $R e_{\lambda}$. The green point corresponds to the measurements by Aliseda an collaborators [1]. (d) Clusters perimeters as a function of the squared root of its area.

complexity of these structures, and the necessity of further studies for better understanding this phenomenon.

\subsection{Conclusions}

Preferential concentration of inertial particles with $\Gamma=1.4$ in the range $2.8<$ $d_{p} / \eta<6.3$ for $340<R e_{\lambda}<810$ and $0.8<S t<4$ has been studied. This is an interesting case as it represents particles in the limit between point and finite size particles.

Clear evidence of clustering was observed. The Voronoï analysis in this case allowed us to easily identify clusters and voids and analyse their structure. Clusters PDFs collapse when they are normalised with their mean value and show a maximum for $A_{C} /<A_{C}>\sim 0.15$. The typical size of clusters, given for instance by the most probable area of clusters, is found to increase from about $20 \eta$ up to $50 \eta$ when the stokes number increases from 1 to 4 . The fractal nature of these structures have been verified, with a complex behaviour that involves many different populations. The cluster properties seems to be qualitatively consistent with the results from [11], studying sub-kolmogorov water droplets in active-grid generated turbulence in a wind tunnel. 
Future studies shall aim at systematically varying $S t$ and $R e_{\lambda}$ independently, to clearly disentangle the specific role of both parameters in clustering properties of inertial particles at moderate density ratio. Comparison with point particle models, including the added mass term, would also be of primary interest to further explore the relevance of those parameters as indicators of clustering.

\section{References}

1. A Aliseda, Alain Cartellier, F Hainaux, and JC Lasheras. Effect of preferential concentration on the settling velocity of heavy particles in homogeneous isotropic turbulence. Journal of Fluid Mechanics, 468(1):77-105, 2002.

2. L Fiabane, R Zimmermann, R Volk, J-F Pinton, and M Bourgoin. Clustering of finite-size particles in turbulence. Physical Review E, 86(3):035301, 2012.

3. Susumu Goto and JC Vassilicos. Self-similar clustering of inertial particles and zeroacceleration points in fully developed two-dimensional turbulence. Physics of Fluids, 18:115103, 2006.

4. Susumu Goto and JC Vassilicos. Sweep-stick mechanism of heavy particle clustering in fluid turbulence. Physical review letters, 100(5):054503, 2008.

5. Arthur La Porta, Greg A Voth, Alice M Crawford, Jim Alexander, and Eberhard Bodenschatz. Fluid particle accelerations in fully developed turbulence. Nature, 409(6823):1017-1019, 2001.

6. Romain Monchaux, Mickaël Bourgoin, and Alain Cartellier. Preferential concentration of heavy particles: A voronoï analysis. Physics of Fluids, 22:103304, 2010.

7. Romain Monchaux, Mickael Bourgoin, and Alain Cartellier. Analyzing preferential concentration and clustering of inertial particles in turbulence. International Journal of Multiphase Flow, 40:1-18, 2012.

8. Nicolas Mordant, Pascal Metz, Olivier Michel, and J-F Pinton. Measurement of lagrangian velocity in fully developed turbulence. Physical Review Letters, 87(21):214501, 2001.

9. Martin Obligado. Fluid-particle interactions: from the simple pendulum to collective effects in turbulence. $\mathrm{PhD}$ thesis, Université de Grenoble, 2013.

10. Nicholas T Ouellette, Haitao Xu, Mickaël Bourgoin, and Eberhard Bodenschatz. Small-scale anisotropy in lagrangian turbulence. New Journal of Physics, 8(6):102, 2006.

11. Sholpan Sumbekova, Alain Cartellier, Alberto Aliseda, and Mickael Bourgoin. Preferential concentration of inertial sub-kolmogorov particles: The roles of mass loading of particles, stokes numbers, and reynolds numbers. Physical Review Fluids, 2(2):024302, 2017.

12. R Volk, N Mordant, G Verhille, and J-F Pinton. Laser doppler measurement of inertial particle and bubble accelerations in turbulence. Europhysics Letters, 81(3):34002, 2008.

13. Romain Volk, R, Delphine Chareyron, and Jean-François Pinton. Mesures d'accélération lagrangienne dans un écoulement anisotrope par vélocimétrie laser doppler étendue. 20ème Congrès Français de Mécanique, 28 août/2 sept. 2011-25044 Besançon, France (FR), 2011.

14. Greg A Voth, Arthur la Porta, Alice M Crawford, Jim Alexander, and Eberhard Bodenschatz. Measurement of particle accelerations in fully developed turbulence. Journal of Fluid Mechanics, 469(1):121-160, 2002.

15. Lian-Ping Wang and Martin R Maxey. Settling velocity and concentration distribution of heavy particles in homogeneous isotropic turbulence. Journal of fluid mechanics, 256:27-68, 1993.

16. G Zocchi, P Tabeling, J Maurer, and H Willaime. Measurement of the scaling of the dissipation at high reynolds numbers. Physical Review E, 50(5):3693, 1994. 